\title{
Vascular Leiomyoma of the Nasal Floor: The Risk of Misdiagnosis
}

\author{
Ki-Il Lee, $\mathrm{MD}, \mathrm{PhD}^{1}$, Hong Geun $\mathrm{An}, \mathrm{MD}^{1}$, Sung Ran Hong, $\mathrm{MD}, \mathrm{PhD}^{2}$, \\ Jong-Yeup Kim, MD, $\mathrm{PhD}^{1}$ and Seung Min In, MD, $\mathrm{PhD}^{1}$ \\ ${ }^{I}$ Department of Otorhinolaryngology-Head and Neck Surgery, Konyang University College of Medicine, Daejeon; and \\ ${ }^{2}$ Department of Pathology, Konyang University College of Medicine, Daejeon, Korea
}

\begin{abstract}
Vascular leiomyoma (VL) is a rare tumor, particularly in the sinonasal region. The etiology of VL is unclear and there are a number of theories regarding how VL develops. To date, approximately 20 cases of VL have been reported in the sinonasal area. This is the first reported case of VL in the right nasal floor. We present the case of an adult patient with mild nasal obstruction and discomfort. Preoperative examination failed to accurately diagnose the patient with VL. Transnasal endoscopic resection was performed. There were no complications or recurrence postoperatively.
\end{abstract}

KEY WORDS: Vascular leiomyoma $\cdot$ Nasal floor $\cdot$ Sinonasal tumor.

\section{INTRODUCTION}

Vascular leiomyoma (VL) or angioleiomyoma is a benign soft-tissue tumor that originates from the vascular smooth muscle. ${ }^{1)}$ In general, this tumor occurs in the lower extremities and the gynecologic region such as the uterus. ${ }^{1-4)} \mathrm{VL}$ has been rarely reported in the head and neck region, particularly the sinonasal area. ${ }^{5)}$ Maesaka et al. ${ }^{6}$ was the first to report an intranasal VL. Our case would be the first case of VL in the right nasal floor in the English literature. This tumor contains of specific histopathologic findings. However, the diagnosis of sinonasal VL preoperatively is challenging. ${ }^{7)}$ We report the clinical and histopathological findings and the possible differential diagnosis of VL located in the right nasal floor of our patient.

\section{CASE REPORT}

A 45-year-old man who experienced mild right nasal obstruction and discomfort was referred from a local ENT clin- ic to our hospital because nasal endoscopy showed a masslike lesion in the right nasal floor. The patient complained of slight unilateral nasal stuffiness but not epistaxis or pain. The mass was located in the anterior portion of the right nasal floor. Nasal endoscopy revealed a broad-based, round, and reddish well-vascularized soft tissue tumor (Fig. 1). Computed tomography (CT) and magnetic resonance imaging (MRI) of the sinuses revealed a well-defined mass 2 $\mathrm{cm}$ in size at the level of the right pyriform aperture, extending to the nasal vestibule. The mass was slightly heterogeneous, enhanced and elevating right inferior turbinate with bony remodeling of right maxilla (Fig. 2). Radiology revealed a solid benign mass appearing like a minor salivary gland tumor such as pleomorphic adenoma or neurogenic tumor (Fig. 3).

Endoscopic removal of the tumor was performed under general anesthesia. The tumor was examined using a 0 - and 30-degree nasal endoscope after shrinking the mucosa with an epinephrine-soaked gauze. It was well-capsulated and adhesion was not noticed. The inside of the mass consisted

Received: November 19, 2019 / Revised: November 28, 2019 / Accepted: December 2, 2019

Address for correspondence: Seung Min In, MD, PhD, Department of Otorhinolaryngology-Head and Neck Surgery, Konyang University College of Medicine, 158 Gwanjeodong-ro, Seo-gu, Daejeon 35365, Korea

Tel: +82-42-600-9215, Fax:+82-42-600-9090, E-mail: ismi96@kyuh.ac.kr 


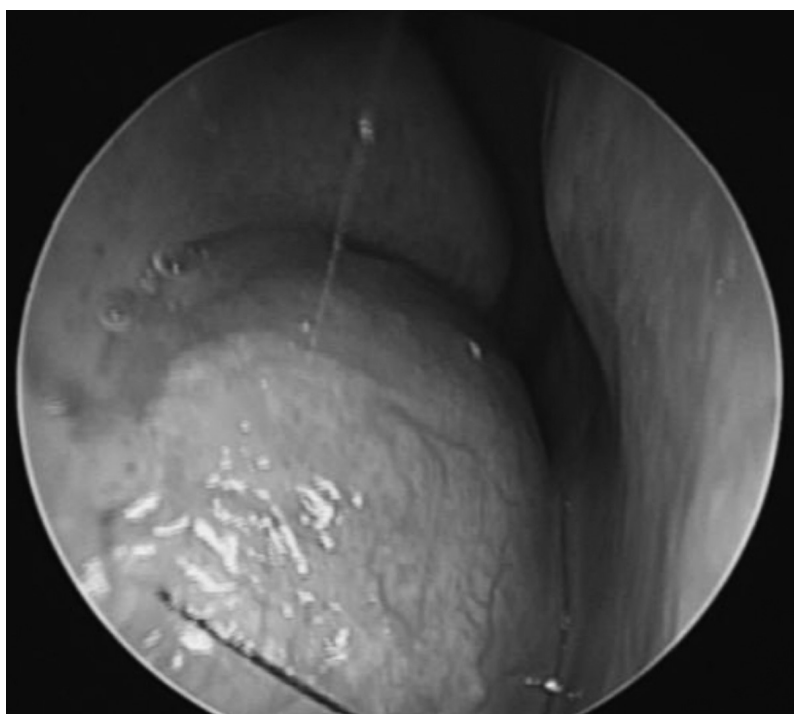

Fig. 1. Endoscopic finding of a soft tissue mass in the right nasal floor. The mass has a round, reddish, and well-vascularized appearance.
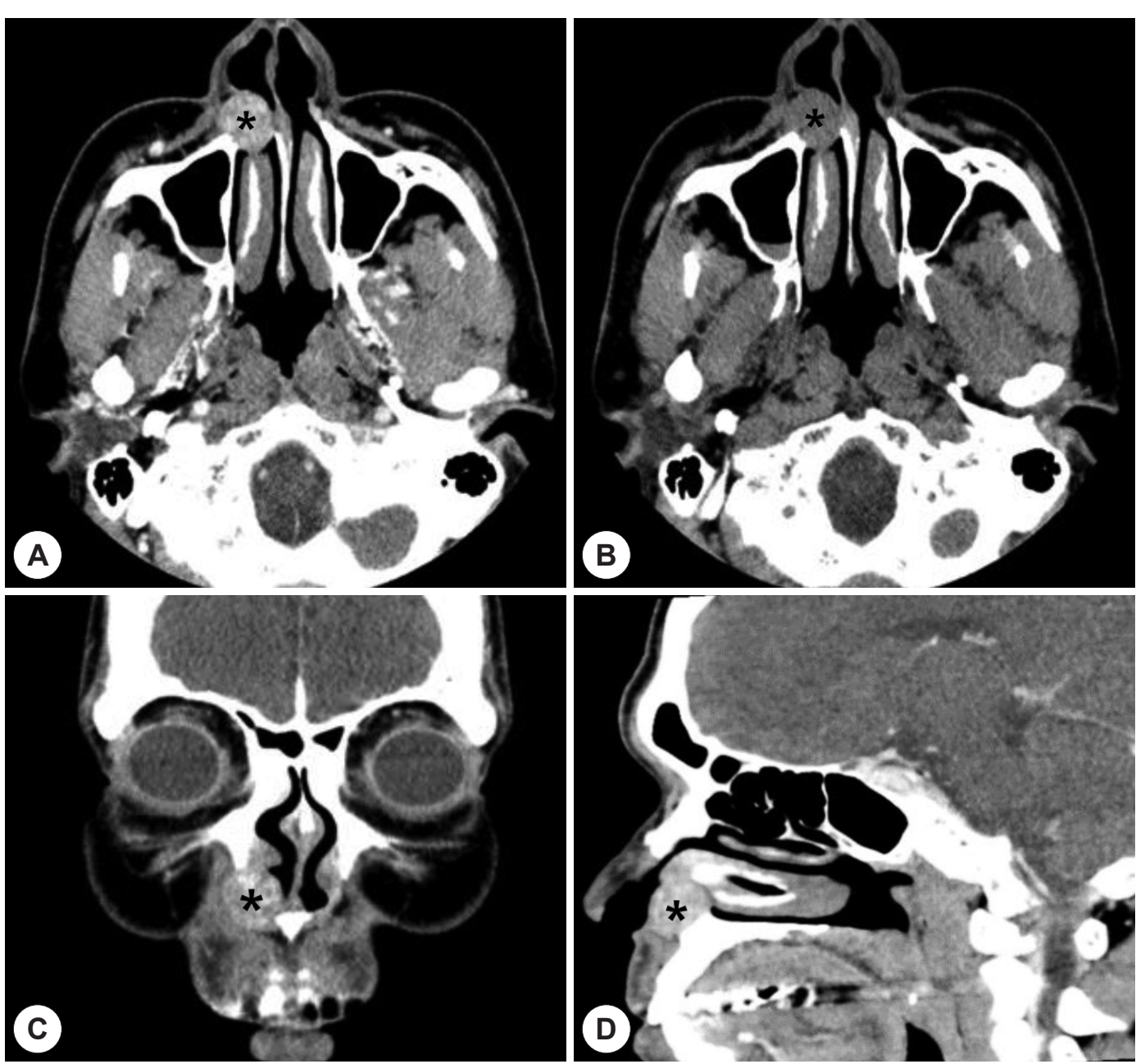

Fig. 2. Preoperative CT scan revealed a slightly heterogenously enhanced mass with bony remodeling in the maxilla. Black asterisk indicates soft tissue density lesion of the right nasal floor. A: enhanced axial. B: non-enhanced axial. C: enhanced coronal. D: enhanced sagittal cut.

of solid components and not cystic fluids. Intraoperative bleeding was mild. The mass was dissected continuously using cold instruments under the guidance of an endoscope (Fig. 4). After the tumor removal, a relatively large space was found in the anterior portion of the right nasal floor and there was no definite bony erosion. The tumor was approximately $1.9 \mathrm{~cm}$ in size. The pathological findings revealed sinonasal VL with growth of smooth muscle cells around the vascular components. Hematoxylin and eosin (H\&E) staining showed a well-demarcated benign spindle cell tumor with irregularly dilated, staghorn-shaped vessels. Immunostaining revealed a diffuse positive reaction for desmin and smooth muscle actin. Masson's trichrome stain also revealed a reddish staining of the spindle cells (Fig. 5). Unilateral nasal obstruction and discomfort were improved immediately after the surgery. The patient has been followed for 4 months with no tumor recurrence (Fig. 6). 

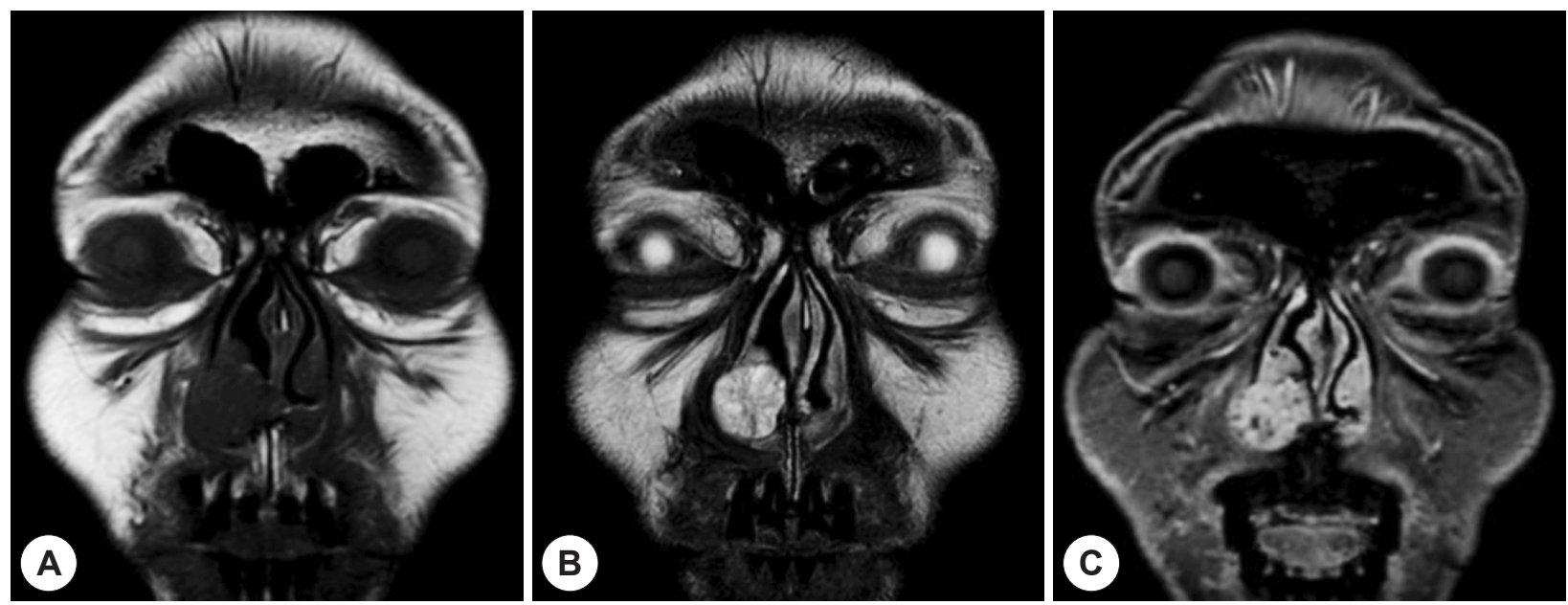

Fig. 3. Preoperative MRI showing the soft tissue tumor in the right nasal floor. A: T1 imaging showing an iso-signal intensity. B: T2 imaging showing mixed low and high signal intensities. C: Tl Gd-enhanced imaging showing a slightly heterogenous enhancement.
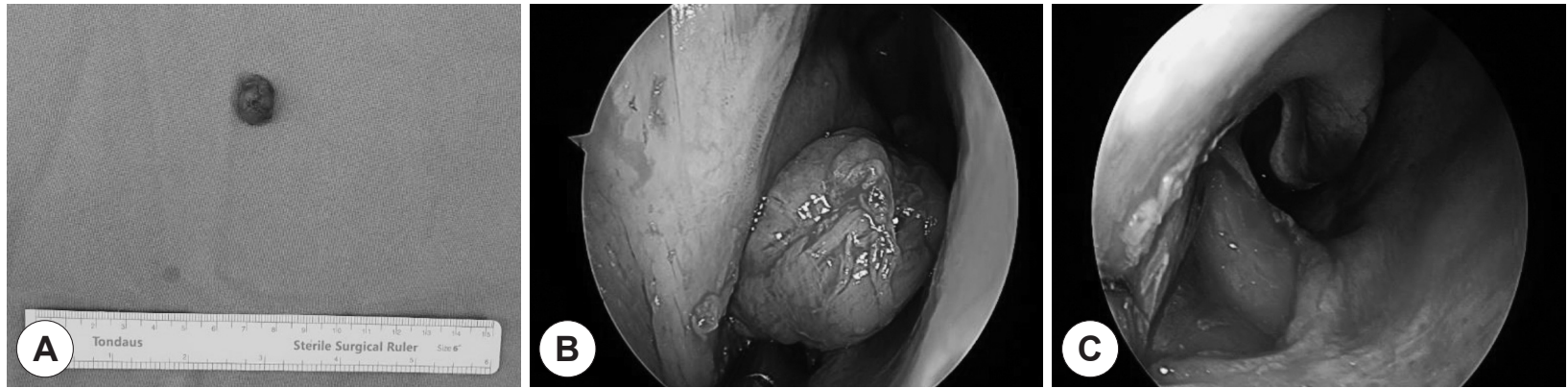

Fig. 4. The excised mass consists of a round, pinkish, and smooth soft tissue. Operative findings showed a well-capsulated soft tissue tumor in the right nasal floor without adhesion or bleeding. A: gross specimen. B: 30-degree endoscope view of the tumor removal. C: 0-degree endoscope view after tumor removal.

\section{DISCUSSION}

VL in the head and neck regions has been rarely reported. ${ }^{58)}$ Moreover, the sinonasal area is an extremely rare location for the occurrence of VL. ${ }^{8)}$ Previous studies reported sinonasal VLs in the inferior turbinate, nasal vestibule, and septum. ${ }^{799)}$ This may be the first reported case of VL in the right nasal floor. Generally, the presenting symptoms are non-specific and depend on the location and size of the tumor. Symptoms of VL include unilateral nasal obstruction, epistaxis, and facial pain. Liu et al. ${ }^{8}$ reported possible causes of pain in patients with VL. Firstly, the contraction of the smooth muscle vessels can cause local ischemia. Secondly, the tumor may compress the surrounding nerves. These two theories explain the effect of the mass. Lastly, secondary inflammation can cause local symptoms. Our patient experienced mild unilateral nasal obstruction and a nonspecific sensation of facial discomfort. The size of the tumor was relatively big but the symptoms were not severe.
Diagnosing sinonasal VL preoperatively is difficult. ${ }^{25)(8)}$ Fine-needle aspiration and cytology cannot provide a definite diagnosis. Imaging techniques such as ultrasonography, $\mathrm{CT}$, and MRI also have no specific features for diagnosing VL. ${ }^{5)}$ In the present case, CT showed a slightly heterogeneously enhanced mass with bony remodeling in the maxilla. MRI showed an iso-signal intensity on T1-weighted images, a slightly heterogeneously enhanced mass on Gadoliniumenhanced images, and a high signal intensity on T2-weighted images. It mimicked a salivary gland or neurogenic tumor during CT and MRI. It was difficult to characterize the tumor type preoperatively.

There are a number of hypotheses for the development of VL. It may be due to vascular malformation or smooth muscle proliferation. ${ }^{9)}$ The most common locations for VL in the sinonasal area are the inferior turbinate, nasal septum, and nasal vestibule. ${ }^{9)} \mathrm{VL}$ in the nasal floor has been rarely reported.

The differential diagnosis of VL includes tumors of mes- 
enchymal origin such as hemangioma, angiofibroma, and neurogenic tumor ${ }^{10)}$ and other benign tumors such as pleomorphic adenoma or cysts. Additionally, we ruled out soft tissue malignancies such as fibrosarcoma, leiomyosarcoma, and neuroectodermal malignancies. ${ }^{11)}$ In our case, radiolog- ic findings suggested pleomorphic adenoma or schwannoma in the sinonasal area. Histopathological examination provides the most accurate diagnosis. Typical findings of H\&E staining include rich vascular channels with thick vessel walls and smooth muscle bundles. ${ }^{5)}$ Focal myxoid change
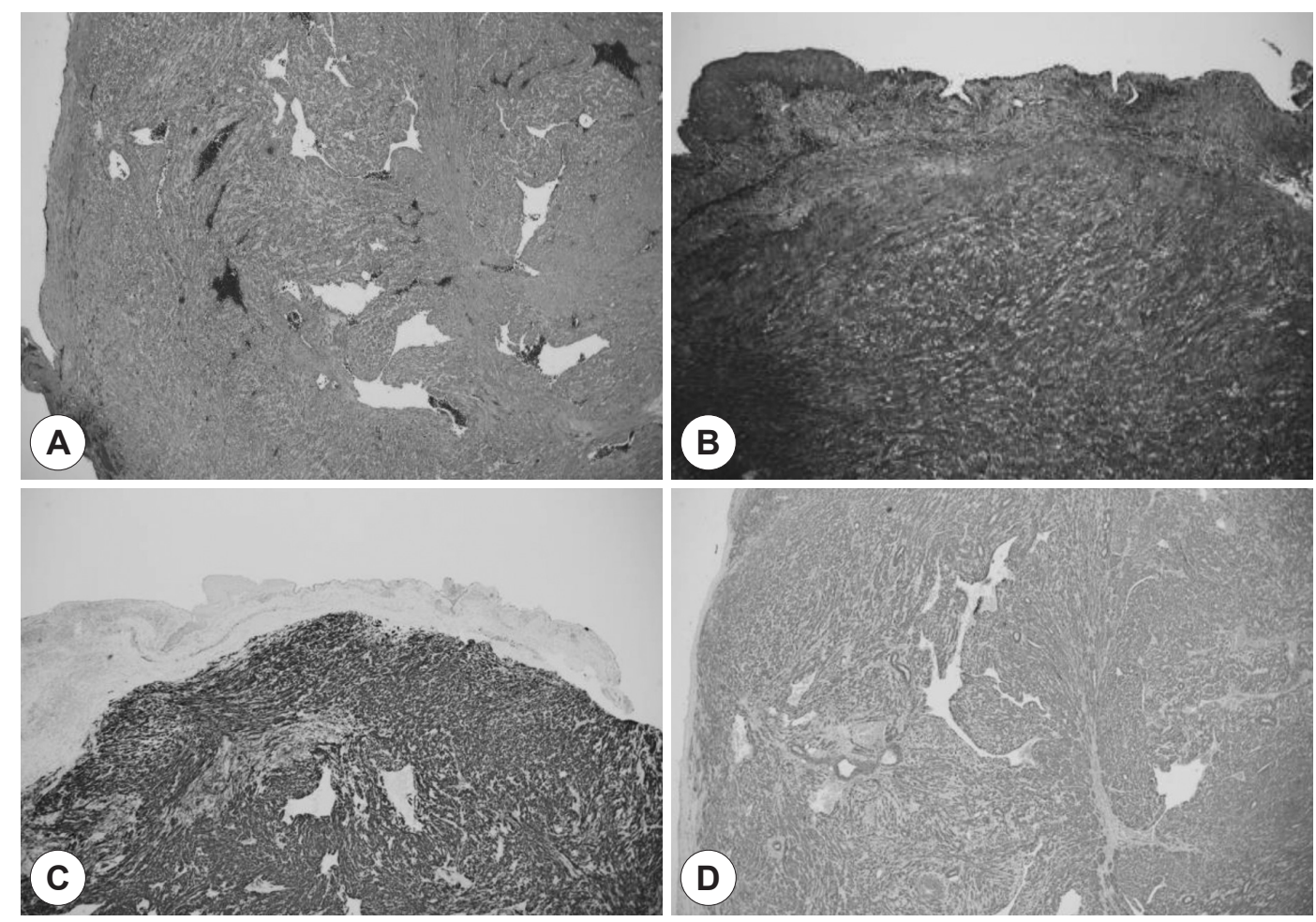

Fig. 5. Pathologic findings. Microscopically, the mass was composed of spindle cells with irregularly dilated, staghorn-shaped vessels. Masson's trichrome staining revealed fuchsinophilia of the spindle cells. Immunohistochemistry revealed a diffuse positive reaction for desmin and a-smooth muscle actin. A: Hematoxylin and eosin stain. B: Masson's trichrome stain. C: Immunohistochemistry for desmin. D: Immunohistochemistry for a-smooth muscle actin.
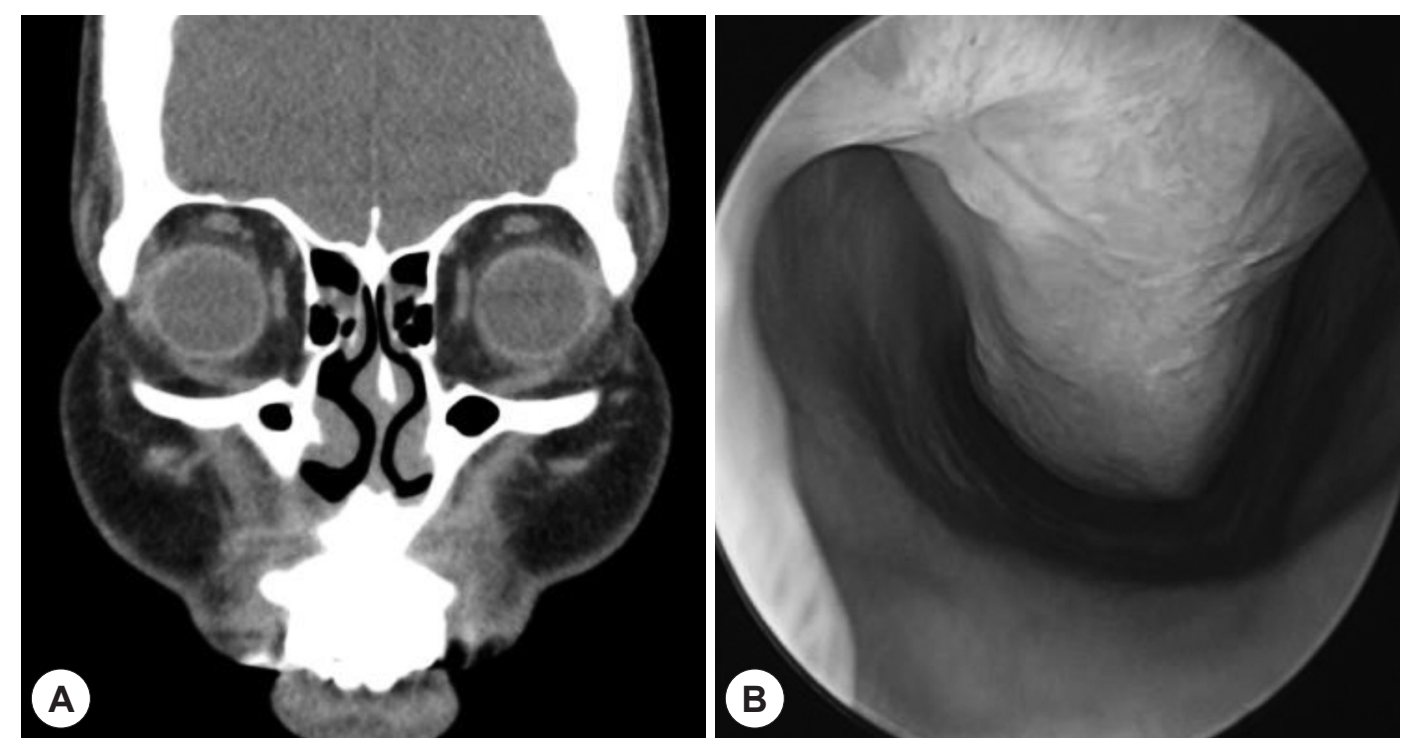

Fig. 6. Postoperative CT and endoscopic finding revealed no enhanced or residual mass in the right nasal floor. A: Enhanced coronal. B: 0-degree endoscope. 
or groups of mature fat cells could also be observed using H\&E staining. ${ }^{9)}$ Masson's trichrome stain, an immunohistochemical stain for smooth muscle cells (actin and desmin) and vascular endothelium cells (CD31, CD34, and factor VIII), could help rule out possible differential diagnoses. ${ }^{912(13)}$ Morimoto ${ }^{3)}$ previously suggested a sub-classification system, which divided VLs into solid, cavernous, and venous types. They reported that these histopathologic subtypes determine the clinical symptoms. In contrast, Yoon et al. ${ }^{7)}$ claimed that the subtypes are not correlated with the clinical manifestations and the prognosis.

Complete surgical resection is the most acceptable treatment. ${ }^{2)}$ Previous studies have suggested the use of enucleation, endoscopic resection, and Caldwell-Luc operation for the treatment of VL in the sinonasal area. ${ }^{1415)}$ The treatment option selected depends on the size, location, and extent of the tumor. The prognosis would be favorable after complete removal of the tumor. No malignant change or recurrence has been reported postoperatively. ${ }^{4}$ In our case, endoscopic transnasal resection was performed and no evidence of recurrence was observed during the 4-month follow-up period.

\section{Acknowledgments}

This work was supported by Konyang University Myunggok Research Fund of 2019.

\section{REFERENCES}

1) Duhig JT, Ayer JP. Vascular leiomyoma. A study of sixtyone cases. Arch Pathol 1959;68:424-30.

2) Hachisuga T, Hashimoto H, Enjoji M. Angioleiomyoma. A clinico- pathologic reappraisal of 562 cases. Cancer 1984;54(1):126-30.

3) Morimoto N. Angiomyoma (vascular leiomyoma): a clinico-pathologic study. Med J Kogoshima Univ 1973;24:663-83.

4) Anderson TD, Weinstein GS. Recurrent angiomyoma (vascular leiomyoma) of the larynx after laser excision. Otolaryngol Head Neck Surg 2000;123(5):646-7.

5) Wang CP, Chang YL, Sheen TS. Vascular leiomyoma of the head and neck. Laryngoscope 2004;114(4):661-5.

6) Maesaka A, Keyaki Y, Nakahashi T. Nasal angioleiomyoma and leiomyosarcoma. Report of two cases. Otologia.(Fukuoka) 1966;12: 42-7.

7) Yoon TM, Yang HC, Choi YD, Lee DH, Lee JK, Lim SC. Vascular leiomyoma in the head and neck region: 11 years experience in one institution. Clin Exp Otorhinolaryngol 2013;6(3):171-5.

8) Liu Y, Li B, Li L, Liu Y, Wang C, Zha L. Angioleiomyomas in the head and neck: A retrospective clinical and immunohistochemical analysis. Oncol Lett 2014;8(1):241-7.

9) Agaimy A, Michal M, Thompson LD, Michal M. Angioleiomyoma of the Sinonasal Tract: Analysis of 16 Cases and Review of the Literature. Head Neck Pathol 2015;9(4):463-73.

10) Ramesh P, Annapureddy SR, Khan F, Sutaria PD. Angioleiomyoma: a clinical, pathological and radiological review. Int J Clin Pract 2004; 58(6):587-91.

11) Brooks JK, Nikitakis NG, Goodman NJ, Levy BA. Clinicopathologic characterization of oral angioleiomyomas. Oral Surg Oral Med Oral Pathol Oral Radiol Endod 2002;94(2):221-7.

12) Gaitan Cepeda LA, Quezada Rivera D, Tenorio Rocha F, Leyva Huerta ER, Mendez Sanchez ER. Vascular leiomyoma of the oral cavity. Clinical, histopathological and immunohistochemical characteristics. Presentation of five cases and review of the literature. Med Oral Patol Oral Cir Bucal 2008;13(8):E483-8.

13) Maeda Y, Hirota J, Osaki T, Hayashi K, Sonobe H, Otsuki Y. Angiomyoma of the upper lip: report of a case with electron microscopic and immunohistochemical observation. Br J Oral Maxillofac Surg 1989;27(3):236-42.

14) Tseng PY, Lai YS, Chen MK, Shen KH. Progesterone receptor expression in sinonasal leiomyoma: a case report and review of the literature. Int J Clin Exp Pathol 2014;7(3):1224-8.

15) Vafiadis M, Kantas I, Panopoulou M, Sivridis E, Exarchakos G. Vascular leiomyoma of the nasal vestibule. Case report and literature review. B-ENT 2008;4(2):105-10. 